\title{
What happens to "useless" natural language mediators?
}

\author{
PHILIP H. MARSHALL and RANDOLPH A. S. SMITH \\ Texas Tech University, Lubbock, Texas 79409
}

\begin{abstract}
Underwood (1972) has hypothesized that natural language mediation may be an epiphenomenon, overloading our conception of human memory. This study examines the specific proposition that natural language mediator (NLM) dropout during learning indicates that they become "useless" and that they are weakened or are replaced by more direct associations. Subjects learned CVC-adjective pairs with the aid of NLMs in a study-test paradigm. After subjects reported that the NLM was no longer needed for response recall ("useless"), they received either 0 or 15 additional study-test sequences of the item. On a later retention test, significant differences were found favoring the 15 additional trials condition for both response and NLM retention. It appears that NLMs do not disappear with disuse, but that they can be long-lasting products of acquisition, potentially serving useful memorial functions.
\end{abstract}

Many studies have purported to demonstrate that verbal mediators generated during acquisition, often called natural language mediators (NLMs), have a definite role in facilitating memorial processes and in improving retention. Adams and Bray (1970) have given NLMs a central role in paired-associate recall, and others have provided evidence for their utility in other paradigms (Marshall \& Smith, in press). Yet with all the data and theorizing, a fundamental question remains.

Underwood (1972) was concerned with the overloading of human memory -with theorists and researchers offering a plethora of mechanisms and devices that potentially may choke the simple elegance of human memorial functioning. One of the culprit mechanisms suggested by Underwood was the NLM. In particular, he reviewed data indicating that, as acquisition trials increase, the frequency of subjects' NLM reporting decreases (Adams \& McIntyre, 1967). Since a report of an NLM operationally indicates that an item is being mediated, he concluded that NLMs become less useful in later acquisition trials, and that subjects may switch to a more efficient rote/associative strategy. This "uselessness," as allegedly evidenced by the dropping out of NLMs, may result from a dispiacement of the NLM in the S-NLM-R chain to where a direct association between the stimulus (S) and the response (R) precedes the occurrence of the NLM. Or, the uselessness may result from the active unlearning of an epiphenomenal implicit response (the NLM) as the criterion response becomes learned.

The authors gratefully acknowledge the assistance of Michael T. Jones and Mark Hannifin in the collection of the data. Reprint requests should be sent to Philip H. Marshall, Department of Psychology, Texas Tech University, Lubbock, Texas 79409.
The design of this study provided for the presentation of additional acquisition trials beyond the trial on which the subjects reported that they no longer needed the NLM to recall the response member of the pair. What happened to the NLMs is described below.

\section{METHOD}

\section{Subjects}

The subjects were 40 Texas Tech University undergraduates participating for course credit.

\section{Materials}

The eight criterion paired-associate items were CVC-adjective pairs from those used by Adams, Marshall, and Bray (1971). The pairs were presented as slides which were automatically advanced by the projector timer. Three-digit number pairs were also used, and these were inserted for study at a point described below.

\section{Procedure}

Subjects were randomly assigned to one of two acquisition conditions. In one condition, subjects received zero additional study-test trials beyond the trial on which the NLM was declared useless. In the other condition, subjects received 15 additional study-test sequences of the items. Before beginning acquisition, subjects were familiarized with methods of producing NLMs and were instructed to generate an NLM for every pair when first presented. On the first study trial each pair was presented until the subject gave an NLM. The first test trial and subsequent study and test trials presented items for $8 \mathrm{sec}$ each. Subjects were told to cease reporting an NLM during the study trials when and if the subject felt that the NLM would no longer be useful during the recall attempt. This procedure defined the drop-out trial. After the test phase of an NLM drop-out trial was over, for the 0 -additional group, that pair was removed from further study and a three-digit number pair was inserted in its place to prevent subjects from intentionally shortening the study list. For the 15-additional group, an additional 15 studytest trials were provided for that item, after which it was removed from the list and a three-digit number pair inserted in its place. Each condition could be considered a correction procedure with irrelevant replacement items. 
All reports of NLMs were oral, and were written down by the experimenter. Different randomized presentation orders were used on each study and test trial. For the 15-additional condition, if the subject returned to reporting an NLM before the end of 15 trials, the counting of the additional trials recommenced at that point. For the retention phase, subjects returned to the laboratory $24( \pm 6) \mathrm{h}$ later. The stimulus terms were presented individually, and the subject was asked to recall first the NLM and then the response for each term. The test stimuli were shown at the rate of one every $15 \mathrm{sec}$.

\section{RESULTS AND DISCUSSION}

Since the treatment for all subjects was the same up to the NLM drop-out trial, one would expect that the drop-out trial would be essentially the same for all subjects. For the 0 -additional condition, the mean dropout trial was 3.14, and for the 15-additional condition it was 3.84-a nonsignificant difference.

On the test portion of the documented drop-out trial, the 0 -additional condition yielded a mean of $85 \%$ correct responses, vs. a mean of $87 \%$ for the 15 -additional condition. This indicates that subjects had a relatively conservative position with respect to the utility of an NLM, and that they "held on" to the NLM until a relatively high level of learning had occurred. In the 15-additional condition, subjects reported NLM usage during the additional trials for $22 \%$ of the total items. It is hard to say whether these were actual required usages of an NLM or whether they were in part inadvertent elicitations of an NLM.

On the retention test the 0 -additional condition yielded a mean of $71 \%$ correct responses compared to a mean of $99 \%$ for the 15 -additional condition. This expected difference in response recall was significant $[t(38)=5.30, p<.001]$. For the purposes of the present study, however, the most telling comparison concerns NLM recall in each condition. The 0 -additional condition resulted in a mean recall of $59 \%$ compared to a mean of $82 \%$ for the 15 -additional condition. This difference was also significant $[\mathrm{t}(38)=3.00, \mathrm{p}<.01]$.

If either the mediational chain had been shortcircuited or the epiphenomenal implicit association had been extinguished, as Underwood (1972) has suggested, one would have expected less correct NLM recall in the 15-additional condition. It appears that additional study trials do not weaken the strength of NLMs, but that they persist and accrue strength beyond the point at which they stop being useful in acquisition. The issue remains, however, whether the persistent NLM represents an inefficient memorial system, or whether it serves some purpose other than facilitating initial acquisition. There is some evidence for the latter in discussions of the role of NLMs (see Adams \& Bray, 1970; Marshall \& Smith, in press). A common theme in these discussions is that the NLM functions in retention much like an information backup system. As such, it provides an alternative means of retrieving a response if a direct association fails, or it serves a confirmatory role and increases response confidence and hence, the vocalization of a response. The results of the present study suggest that NLMs do not "go away" with disuse, but rather that they can be relatively long-lasting and useful products of acquisition. To refer to a well known example, we may find it very easy to automatically and mechanically recite the lines of the musical staff $(E, G, B, D, F)$, but we have to admit that we experience equal ease in remembering the "NLM" that helped us learn it in the first place (Every Good Boy Does Fine).

\section{REFERENCES}

Adams, J. A., \& Bray, N. W. A closed-loop theory of paired-associate verbal learning. Psychological Review, 1970, 77, 385-405.

adams, J. A., Marshall, P. H., \& Bray, N. W. Closed-loop theory and long-term retention. Journal of Experimental Psychology, 1971, 90, 242-250.

Adams, J. A., \& McIntyre, J. S. Natural language mediation and all-or-none learning. Canadian Journal of Psychology, 1967, 21, 436-449.

Marshall, P. H., \& Smith, R. A. S. Verification processes in recognition memory: The role of natural language mediators. British Journal of Psychology, in press.

UNDERWOOD, B. J. Are we overloading memory? In A. W. Melton \& E. Martin (Eds.), Coding processes in human memory. Washington, D.C: V. H. Winston, 1972.

(Received for publication April 21, 1977.) 